\title{
Sexual Harassment in Psychiatry Residency: a Call to Action
}

\author{
Brianna Williamson ${ }^{1}$ (B)
}

Received: 5 March 2021 / Accepted: 25 May 2021 / Published online: 25 June 2021

(C) Academic Psychiatry 2021

Sweetie, Love, Nurse - these are titles all too familiar to female-presenting physicians. Often, they are used by patients and their families. Is this sexual harassment or just stereotyping? As we live into the \#MeToo (and \#MeToo Medicine) era and own our voices, it is time to consider the power of words and titles - their power to both build and tear down. "Sweetie" is a title I have heard often and, depending on the context and accompanying gestures and glances, it ranges from a nonissue, to confusing, to deeply uncomfortable. Whether the response is outrage, acceptance, or somewhere in between, trainees need safe spaces to express a range of reactions and garner support. Otherwise, trainees, members of a vulnerable population themselves, are left alone to determine how to react to the many forms of discrimination we encounter. One more small weight to add to the backpack women physicians wear too often in silence.

In June 2018, the National Academies of Sciences, Engineering, and Medicine (NASEM) published a report on sexual harassment, revealing that the prevalence of sexual harassment in academic medicine is about double that of other science and engineering specialties. According to their calculations, half of all trainees at the institutions they surveyed reported sexual harassment from staff or faculty [1]. A metaanalysis of 51 studies demonstrated similar findings, indicating that nearly $60 \%$ of trainees experienced some type of harassment over the course of their medical training [2]. While these results are startling, they were limited in scope because the study did not include all people trainees interact within a professional setting.

In the June 2018 study, NASEM employed a broad definition of sexual harassment, breaking it into three categories: first, gender harassment, defined as sexist hostility or crude behavior; second, unwanted sexual attention, defined as unwelcome verbal or physical advances; and third, sexual

Brianna Williamson

bwilliamson@psychiatry.arizona.edu

1 University of Arizona, Tucson, AZ, USA coercion, defined as favorable professional or educational treatment dependent upon sexual activity [1]. When I consider my short time in residency, I have, myself, experienced the first two on multiple occasions. However, it is not at the hands of my superiors; it is from patients. This makes me question if it even "counts." My patients are sick and not always fully aware of what they say and how it sounds. Thus, I have tended to blame myself for not knowing how to best act, or what exactly to say. Preliminary research indicates this is a common experience; $90 \%$ of female physicians report unwanted sexual attention from patients and/or patient families [3].

Many factors contribute to trainees' reluctance to speak out about these experiences. Foremost is our inexperience - many of us are new at navigating complex working situations. For many of my colleagues, residency is their first "real job," and few relish the thought of being labeled "difficult" or "sensitive" by speaking out. These factors, combined with few established reporting mechanisms for physician sexual harassment by patients, foster a culture of silence. However, female colleagues continue to share concerns, frustrations, and stories of their own between didactic sessions and in on-call rooms across the country.

Just like many psychiatry trainees, I entered the field because I felt a strong desire to work with and advocate for patients, especially those with mental illness. However, as I gain more experience, I am convinced that we can better advocate for residents as we navigate the complexities of unwelcome advances and commentary. What inherent tools (or tools taught in training) can we use to maneuver these difficult situations and maintain therapeutic alliance with patients?

In their 2019 publication, Goldenberg et al. proposed a framework to address trainee mistreatment called ERASE. It focused on five core elements: (1) expect that mistreatment will happen, (2) recognize when it does occur, (3) address the situation in real time, (4) support the trainee after the event, and (5) establish a positive culture [4]. This notable framework begins with expectation; it implores supervisors to expect and, perhaps just as importantly, recognize this behavior. This expectation and realization are paramount to addressing harassment in a productive fashion. Unfortunately, 
expectation and realization require effort and education of both residents and supervisors, including those who do not fall into the harassed group.

More and more, institutions and programs are broadening their policies to include improved diversity and inclusion and wellness practices. What a great opportunity to prioritize gender-based antidiscrimination tactics for resident and faculty development. Though less common, it is also crucial to recognize that harassment also happens to male-presenting residents. In an attempt to protect all trainees, I propose a top-down approach to best protect trainees and foster an environment that guides and supports the next generation of physicians. Any environment successful in promoting antidiscrimination policies must rely on institutional, departmental, and programmatic support. This not only requires protected time and trainings for supervisors, but it also requires meaningful and open conversation between trainees and supervising attendings about the frequency of gender-based discrimination. What better time to do right by our trainees than now? Our focus must be on how to best support physicians by addressing the unfortunately common experience of sexual discrimination for residents. The weight of discrimination in the backpack is surely more manageable when acknowledged, addressed, and shared.

\section{Declarations}

Disclosures The author states that there is no conflict of interest.

\section{References}

1. Johnson PA, Widnall SE, Benya FF. National Academies of Sciences, Engineering, and Medicine. In: Sexual harassment of women. Washington, DC: National Academies Press; 2018.

2. Fnais N, Soobiah C, Chen MH, Lillie E, Perrier L, Tashkhandi M, et al. Harassment and discrimination in medical training: a systematic review and meta-analysis. Academic Medicine: journal of the Association of American Medical Colleges. 2014;89(5):817-27.

3. Mathews E, Hammarlund R, Kullar R, Mulligan L, Le T, Lauve S, et al. Sexual harassment in the house of medicine and correlations to burnout: a cross-sectional survey. Ochsner J. 2019;19(4):329-39.

4. Goldenberg MN, Cyrus KD, Wilkins KM. ERASE: a new framework for faculty to manage patient mistreatment of trainees. Acad Psychiatry. 2019;43:396-9.

Publisher's Note Springer Nature remains neutral with regard to jurisdictional claims in published maps and institutional affiliations. 